\title{
A Word From the Editor
}

I'm very pleased to bring this issue of the TESL Canada Journal to your attention. It contains thought-provoking articles and book reviews on important topics in our field.

Lisa Leopold's corpus-based research focuses on strategies for making email requests in the North American business context. The findings from the authentic data that she collected and analyzed will be of particular interest to instructors of pragmatics and those preparing ESL learners for professional e-mail communication. Maxime Lavallée and Kim McDonough report the results of a study that compared ratings of writing prompts for either a cause or an effect essay based on source texts. Holistic ratings were assigned to the compositions, and lexical features of the source texts and compositions were then examined. The findings of their study will be relevant to ESL writing instructors and assessors. Ji Hye Shin and Peggy Albers explore the differential effects of the Cyber Home Learning System used to enhance the English language proficiency of middle school students in South Korea. Their recommendations will be valuable for practitioners using online learning programs for English language learners in a variety of contexts.

The first "In the Classroom" article, by Kim McDonough and César García Fuentes, explores the language used by EFL university students in task-based activities. Students wrote cause/effect and problem/solution paragraphs, individually or collaboratively, and these were rated and analyzed for grammatical structure and accuracy. The results offer useful implications for English language writing instructors. Nikolay Slavkov's "In the Classroom" article provides a stimulating explanation of the features of Google Drive that can be used to enhance writing instruction, as well as clear directions and examples of ways in which it can be used online or in computer labs. This article will be of value to both novices and experts interested in promoting computermediated writing instruction with the use of the Google Drive app.

In his "Perspectives" article, John Walker reports the results of a study in which the Critical Incident Technique (CIT) was used to assess student satisfaction with the services provided by their ESL program. The participants recorded two critical incidents, one positive and one negative. This qualitative approach provides rich data for identifying the services most relevant to ESL learners and for enhancing the quality of second language programs; it should be of interest to all ESL program administrators and instructors.

We present reviews of three books in this issue that will be of interest to ESL researchers and/or instructors. The first is A Scholar's Guide to Getting Published in English: Critical Choices and Practical Strategies (2013), by Mary Jane Curry and Theresa Lillis. Ismaeil Fazel offers his perspective on the sug- 
gestions provided for emerging scholars interested in writing for publication. Jennifer Foote reviews Introducing Second Language Acquisition: Perspectives and Practices (2014) by Kirsten Hummel and finds it reader-friendly, with a rich list of resources for further reading and viewing. Finally, An Introduction to English Sentence Structure: Clauses, Markers, Missing Elements (2014) by Jon Jonz is recommended by Karin Keefe as an instructional resource or a selfstudy text for readers with a limited knowledge of functional grammar. We hope that these reviews will provide useful suggestions for your professional development, either individually or in a reading group with colleagues in your ESL program.

We would like to acknowledge our many supporters who have contributed their time and expertise to the TESL Canada Journal over the years. Our sincere thanks go to those retiring from our review board in summer 2015: Patricia Balcom (Université de Moncton), Averil Coxhead (Wellington), Ellen Cray (Carleton University), Alister Cumming (OISE, University of Toronto), Patricia Duff (University of British Columbia), Jim King (University of Leicester), Paula Kristmanson (University of New Brunswick), Hossein Nassaji (University of Victoria), and Kelleen Toohey (Simon Fraser University).

We would like to welcome several new members to the TESL Canada Journal review board: Pauline Foster (St. Mary's University, London), Kim McDonough (Concordia University), Bonny Norton (University of British Columbia), Leila Ranta (University of Alberta), and Stuart Webb (University of Western Ontario). We look forward to working with you in the coming years.

To our ongoing committee and board members and staff, thank you for your continuing support of the TESL Canada Journal. Our next issue, Language Assessment in Canada: Critical Issues and Research Agenda, is guest edited by Dr. Liying Cheng, Professor of Language Assessment and Evaluation at Queen's University. It should be online in the next few months and will be of interest to all ESL instructors, administrators, and assessors.

Marian Rossiter 


\section{Un mot de l'éditrice}

Il me fait grand plaisir d'attirer votre attention vers ce numéro de la Revue TESL Canada. Il présente des articles qui suscitent la réflexion et des critiques de livres portant sur des sujets importants dans notre domaine.

La recherche de Lisa Leopold repose sur un corpus et porte sur les stratégies visant les demandes par courrier électronique dans le contexte du monde des affaires en Amérique du Nord. Les résultats des données authentiques qu'elle a recueillies et analysées intéresseront ceux qui enseignent la compétence pragmatique et ceux qui forment les étudiants en ALS en communication professionnelle par courriel. Maxime Lavallée et Kim McDonough font état des résultats d'une étude ayant comparé les évaluations de tâches d'écriture pour des rédactions portant sur les causes ou bien sur les effets et basées sur des textes sources. On a attribué des évaluations globales aux rédactions et ensuite examiné les caractéristiques lexicales des textes sources et des rédactions. Les résultats de leur étude seront pertinents pour les enseignants et évaluateurs en ALS. Ji Hye Shin et Peggy Albers explorent les effets différentiels du Cyber Home Learning System visant l'amélioration de la compétence langagière en anglais d'élèves de l'intermédiaire en Corée du Sud. Leurs recommandations s'avéreront précieuses aux praticiens qui emploient les programmes d'apprentissage en ligne avec des apprenants d'anglais dans divers contextes.

Le premier article dans la section «En Salle de classe » est par Kim McDonough et César García Fuentes qui explorent la langue d'emploient les étudiants en ALE à l'université lors d'activités basées sur la participation. Les étudiants ont rédigé des paragraphes cause-effet et problème-solution, seul ou en groupes. Ceux-ci ont été évalués et analysés en fonction de la structure grammaticale et l'exactitude. Les résultats offrent des implications utiles aux enseignants de l'écriture en anglais. Dans son article, Nikolay Slavkov propose une explication intrigante des fonctionnalités de Google Drive qui peuvent appuyer l'enseignement de l'écriture, et l'accompagne de directives et d'exemples clairs portant sur des façons d'intégrer celles-ci en ligne ou en laboratoire informatique. Cet article sera utile tant aux débutants qu'aux experts qui s'intéressent à la promotion de l'enseignement de l'écriture assisté par ordinateur avec l'application Google Drive.

L'article de John Walker dans la section "Perspectives" rend compte d'une étude qui a employé la méthode des incidents critiques pour évaluer la satisfaction qu'éprouvent les étudiants face aux services fournis par leur programme d'ALS. Les participants ont fait part de deux incidents critiques, dont un positif et un négatif. Cette approche qualitative offre une abondance de données pour l'identification des services les plus pertinents aux appre- 
nants en ALS et les plus aptes à améliorer la qualité des programmes d'ALS. Les administrateurs et les enseignants en ALS y trouveront surement leur compte.

Nous présentons des critiques de trois livres dans ce numéro; celles-ci ne manqueront pas d'intéresser les chercheurs et les enseignants en ALS. La première critique porte sur A Scholar's Guide to Getting Published in English: Critical Choices and Practical Strategies (2013), écrit par Mary Jane Curry et Theresa Lillis. Ismaeil Fazel offre sa perspective sur les suggestions qu'on y émet pour les nouveaux chercheurs voulant se faire publier. Dans son compterendu de Introducing Second Language Acquisition: Perspectives and Practices (2014) par Kirsten Hummel, Jennifer Foote nous indique que le livre est d'une grande lisibilité et qu'il présente une liste approfondie de ressources pour poursuivre notre lecture. Finalement, Karin Keefe recommande An Introduction to English Sentence Structure: Clauses, Markers, Missing Elements (2014) par Jon Jonz comme ressource pédagogique ou manuel pour autodidactes ayant une connaissance limitée de la grammaire fonctionnelle. Nous espérons que ces comptes rendus vous serviront de guides dans votre développement professionnel, soit à titre individuel, soit comme groupe de lecture avec les collègues de votre programme en ALS.

Nous tenons à reconnaitre tous ceux et celles qui nous ont appuyé et qui ont consacré temps et expertise à la Revue TESL Canada au fil des années. Nous adressons nos plus sincères remerciements à ceux et celles qui ont quitté le comité de révision à l'été 2015: Patricia Balcom (Université de Moncton), Averil Coxhead (Wellington), Ellen Cray (Université Carleton), Alister Cumming (OISE, Université de Toronto), Patricia Duff (Université de la Colombie-Britannique), Jim King (University of Leicester), Paula Kristmanson (Université du Nouveau-Brunswick), Hossein Nassaji (Université de Victoria), and Kelleen Toohey (Université Simon Fraser).

Nous souhaitons la bienvenue à plusieurs nouveaux membres de notre comité de révision: Pauline Foster (St. Mary's University, London), Kim McDonough (Université Concordia), Bonny Norton (Université de la ColombieBritannique), Leila Ranta (Université de l'Alberta) et Stuart Webb (University of Western Ontario). Nous avons hâte de travailler avec vous à l'avenir.

À notre personnel et aux membres de nos comités en place, un grand merci pour votre soutien continu. Dr. Liying Cheng, professeur en évaluation des langues à l'Université Queen, sera le rédacteur invité de notre prochain numéro, Language Assessment in Canada: Critical Issues and Research Agenda. Ce numéro, qui devrait être en ligne d'ici quelques mois, présentera un intérêt certain pour tous les enseignants, administrateurs et évaluateurs en ALS.

Marian Rossiter 